\title{
Deriving the mass distribution of M87 from globular clusters
}

\author{
Xiaoan $\mathrm{Wu}$ and Scott Tremaine \\ Princeton University Observatory, Peyton Hall \\ Princeton, NJ 08544-1001, USA \\ xawn@astro.princeton.edu, tremaine@astro.princeton.edu
}

\begin{abstract}
We describe a maximum-likelihood method for determining the mass distribution in spherical stellar systems from the radial velocities of a population of discrete test particles. The method assumes a parametric form for the mass distribution and a non-parametric distribution function. We apply the method to a sample of 161 globular clusters in M87. We find that the mass within $32 \mathrm{kpc}$ is $2.3 \pm 0.7 \times 10^{12} \mathrm{M}_{\odot}$, and the exponent of the density profile $\rho \propto r^{-\alpha}$ within $7-110$ $\mathrm{kpc}$ is $\alpha=1.6 \pm 0.4$. The anisotropy of the globular-cluster velocity distribution cannot be determined reliably with the present data.
\end{abstract}

Subject headings: Methods: statistical - Galaxy: kinematics and dynamics Galaxy: fundamental parameters - Galaxy: individual (M87) - Galaxy: globular clusters: general

\section{Introduction}

The mass distribution in elliptical galaxies is difficult to measure, in part because ellipticals do not contain gas or star disks like those found in spiral galaxies. The mass distribution is particularly uncertain beyond the effective radius, where the surface brightness fades rapidly. Fortunately, globular clusters (GCs) are found at large distances in many elliptical galaxies. For example, more than $8 \times 10^{3} \mathrm{GCs}$ are believed to be present in M87 outside 3 times the effective radius $r_{e} \sim 7 \mathrm{kpc}$ (McLaughlin 1999a; Zeilinger, Møller \& Stiavelli 1993). GCs in nearby galaxies provide relatively bright pointlike sources for which radial velocities can be determined. By analyzing the statistics of the positions and radial velocities of GCs, we may constrain the mass profiles of their host galaxies and explore the dark matter distribution in the outer parts of elliptical galaxies. Planetary nebulae also provide excellent kinematic probes for similar reasons (e.g. Douglas et al. 2002). 
The database of extragalactic GCs with radial velocities has been steadily growing in recent years. The most intensively observed systems are NGC 1399 (Dirsch et al. 2004) and M87. In this paper we examine the latter system. Harris (1986) and McLaughlin (1999a) observed the density profile of GCs in M87 out to $110 \mathrm{kpc}$. Mould et al. (1990) compiled a list of 43 GCs with radial velocities, from which Merritt \& Tremblay (1993) derived a mass of $5-10 \times 10^{12} \mathrm{M}_{\odot}$ within $50 \mathrm{kpc}$. They also estimated that about 200 and $1000 \mathrm{GCs}$ would be required to derive accurately the slope of the mass profile of the galaxy and the velocity anisotropy of the GCs, respectively. Cohen and Ryzhov (1997) and Cohen (2000) obtained radial velocities for 221 GCs. This dataset was used to study the spin (Cohen and Ryzhov 1997; Cohen, Blakeslee \& Ryzhov 1998; Kissler-Patig \& Gebhardt 1998) and mass profile of M87 (Romanowsky \& Kochanek 2001).

Although there is significant rotation in the M87 GC system, the fractional kinetic energy in rotation $(\Omega R / \sigma)^{2} \sim 20 \%$ is relatively small, so it should be safe to ignore rotation in our analysis and adopt the assumption that the system is spherical (Côté et al. 2001). Thus the data pairs $\left[R, v_{z}\right]$ contain all available dynamical information, where $R$ is the projected distance from the GC to the center of the galaxy, and $v_{z}$ is its velocity in the line of sight. To convert angular distance to physical distance, we adopt a distance to M87 of $16.3 \mathrm{Mpc}$ (i.e. 79 pc $\operatorname{arcsec}^{-1}$ ) following Cohen (2000).

Maximum likelihood analysis provides a powerful tool to analyze discrete data. It has been used to derive the mass distribution of spherical galaxies by Merritt (1993) and Merritt \& Saha (1993). Our method for deriving spherical mass distributions from discrete data is reviewed in Section 2. We use simulated data to test our method in Section 3. In Section 4 we constrain the mass profile of M87 and the distribution function (hereafter DF) of its GCs. We discuss our results in Section 5.

\section{Methods}

Assume that $B_{r}$ is a set of mutually exclusive and exhaustive hypotheses, $H$ is all available prior information, and $A$ is some observed event. We quote Kendall, Stuart and Ord (1987) with minor changes in notation: "We may write Bayes' theorem in the form

$$
P\left(B_{r} \mid A, H\right) \propto P\left(B_{r} \mid H\right) L H\left(A \mid B_{r}, H\right)
$$

... The principle of maximum likelihood states that, when confronted with a choice of hypotheses $B_{r}$ we choose that one (if any) which maximizes $L H$. In other words, we are to choose that hypothesis which gives the greatest probability to the observed event. Whereas Bayes' theorem enjoins the maximization of the joint probability of $B_{r}$ and $A$, maximum 
likelihood requires the maximization of the conditional probability of $A$ given $B_{r}$." The quantities $P\left(B_{r} \mid A, H\right)$ are posterior probabilities, $P\left(B_{r} \mid H\right)$ are prior probabilities, and $L H$ is likelihood. The maximum likelihood method states that the prior probability should be assumed the same for all hypotheses if there is no prior information available. We now apply these concepts to constrain the potential $\Phi$ from discrete data pairs $\left[R_{i}, v_{z i}\right]$.

In a spherical system, the DF depends on at most two integrals of motion, energy $E$ and scalar angular momentum $L$, and thus takes the form $f(E, L)$. We normalize so that

$$
\int f(E, L) d^{3} \mathbf{x} d^{3} \mathbf{v}=1
$$

We introduce a Cartesian coordinate system $(x, y, z)$ with origin at the galactic center, and $z$-axis along the line of sight. In this system, the projected radius is

$$
R=\sqrt{x^{2}+y^{2}}
$$

The probability that a given GC is found in the interval $d R d v_{z}$ is $2 \pi R g\left(R, v_{z}\right) d R d v_{z}$, where

$$
g\left(R, v_{z}\right)=\int f(E, L) d z d v_{x} d v_{y}
$$

Because of spherical symmetry, we may assume without losing any information that all GCs lie in the $x-z$ plane and $x>0$. Thus we have

$$
\begin{aligned}
E & =\Phi(r)+\frac{1}{2}\left(v_{x}^{2}+v_{y}^{2}+v_{z}^{2}\right) \\
L & =\sqrt{\left(v_{z} R-v_{x} z\right)^{2}+v_{y}^{2}\left(R^{2}+z^{2}\right)}
\end{aligned}
$$

For continuous data, we can in principle obtain a unique $\operatorname{DF} f(E, L)$ and potential $\Phi$ from equation (4) (Merritt 1993, and references therein), although the inversion process amplifies any noise that is present in the data. For a set of discrete data pairs $\left[R_{i}, v_{z i}\right]$, we need to use statistical methods. Given a potential $\Phi(r)$ and a DF $f(E, L)$, the likelihood of the observation is

$$
\begin{aligned}
L H(\Phi, f) & \equiv \operatorname{LH}\left(\left[R_{i}, v_{z i}\right] \mid \Phi, f\right) \\
& =\prod_{i} g\left(R_{i}, v_{z i} \mid \Phi, f\right) \cdot 2 \pi R_{i} \\
& \propto \prod_{i} g\left(R_{i}, v_{z i} \mid \Phi, f\right) .
\end{aligned}
$$

The maximum likelihood method assumes that the best estimate of the potential and DF is the one that maximizes $L H(\Phi, f)$. More generally, Bayes' theorem states that if we know 
some prior information $H$ which gives a prior probability $P(\Phi, f \mid H)$, we should maximize the posterior probability $P(\Phi, f \mid H) L H(\Phi, f)$ rather than the likelihood.

This formalism can easily be generalized to account for selection effects and observational errors in radial velocities. For example, in most surveys we observe GCs only in a limited range of radii $\left(R_{s 0}<R<R_{s 1}\right)$. To account for this limitation, we simply modify the normalization to require that

$$
\int_{R_{s 0}}^{R_{s 1}} g\left(R, v_{z}\right) 2 \pi R d R \int d v_{z}=1 .
$$

To account for the errors in radial velocities, we just need to calculate $L H$ with $g\left(R, v_{z}\right)$ replaced by

$$
g^{\prime}\left(R, v_{z}\right)=\frac{1}{\sqrt{2 \pi} \sigma_{v}} \int g\left(R, v_{z}\right) e^{-\left(v-v_{z}\right)^{2} / 2 \sigma_{v}^{2}} d v
$$

Here we have assumed that the error $\sigma_{v}$ is the same for all GCs, although the method is easily generalized to individual measurement errors. Note that our method also probes, though with less power, the potential beyond the maximum survey radius $R_{s 1}$, both because we observe the projected distribution, which includes GCs with radial distances to the galactic center larger than $R_{s 1}$ and because some GC orbits have apocenters outside $R_{s 1}$.

In principle, all physically possible potentials and DFs should be explored to maximize $L H(\Phi, f)$. The most general way to do so is to assume non-parametric forms for both potentials and DFs, but this is cumbersome and computationally expensive. Instead we optimize within a family of parametrized potentials: we assume an analytical form $\Phi(r, \mathbf{X})$ and infer the parameters $\mathbf{X}$ (which can be viewed as a row vector). For example, we may assume a power-law density-potential pair

$$
\begin{aligned}
\rho(r) & =\rho_{0}\left(\frac{r}{r_{0}}\right)^{-\alpha} \\
\Phi(r) & =\frac{4 \pi G \rho_{0} r_{0}^{2}}{(2-\alpha)(3-\alpha)}\left(\frac{r}{r_{0}}\right)^{2-\alpha} \quad(\alpha<3),
\end{aligned}
$$

where $r_{0}$ is an arbitrary radius, and then $\mathbf{X}=\left\{\rho_{0}, \alpha\right\}$. We also investigate the Navarro, Frenk \& White (1997) density-potential pair,

$$
\begin{aligned}
\rho(r) & =\frac{\rho_{0}}{\frac{r}{r_{c}}\left(1+\frac{r}{r_{c}}\right)^{2}}, \\
\Phi(r) & =-4 \pi G \rho_{0} r_{c}^{2} \frac{\ln \left(1+\frac{r}{r_{c}}\right)}{\frac{r}{r_{c}}} .
\end{aligned}
$$


Here $r_{c}$ is the concentration radius and $\mathbf{X}=\left\{\rho_{0}, r_{c}\right\}$.

If the DF $f(E, L)$ were known, the problem of optimizing the parameters $\mathbf{X}$ would be easy to solve. We would calculate $L H$ as a function of $\mathbf{X}$ and figure out where $L H$ peaks. However, in practice the DF is an unknown function, and we have to derive the DF and $\mathbf{X}$ simultaneously. This can be done by parametric or non-parametric methods.

In the parametric method (Merritt \& Saha 1993), we choose a limited number of basis functions $f_{k}(E, L)$ (i.e. a complete set of basis functions truncated to some order $N$ ), which are smooth in phase space. Thus, the DF is approximated as

$$
f(E, L)=\sum_{k} w_{k} f_{k}(E, L), \quad k=1, \ldots, N .
$$

This method ensures that the derived DF is smooth; however, it is difficult to ensure that $f(E, L) \geq 0$ for all $(E, L)$ since neither $w_{k}$ nor $f_{k}$ is necessarily non-negative. Furthermore, if $N$ is small we cannot ensure that equation (11) gives a good approximation to the actual DF. Of course, we can always increase $N$ to improve the approximation, but then we cannot ensure that the best-fit DF will be smooth.

In the non-parametric method (Merritt 1993), we divide the $E-L$ space into $N_{E} \times N_{L}$ bins, which are denoted by the double index $m n, m=1, \ldots, N_{E}, n=1, \ldots, N_{L}$. Notice that the DF is isotropic if $N_{L}=1$ and otherwise may be anisotropic. Then we construct a set of top-hat functions,

$$
h_{m n}(E, L)=\left\{\begin{aligned}
V_{m n}^{-1} & \text { if } V_{m n} \neq 0 \text { and }(E, L) \in \text { bin } m n \\
0 & \text { otherwise }
\end{aligned}\right.
$$

where $V_{m n} \equiv \int_{\text {bin } m n} d^{3} \mathbf{x} d^{3} \mathbf{v}$ is the phase-space volume within the survey limits that is associated with bin $m n$. Thus, we have

$$
\begin{aligned}
& \int h_{m n}(E, L) d^{3} \mathbf{x} d^{3} \mathbf{v}=1, \\
& f(E, L)=\sum_{m, n} w_{m n} h_{m n}(E, L), \\
& f_{m n}=\left\{\begin{aligned}
w_{m n} / V_{m n} & \text { if } V_{m n} \neq 0 \\
0 & \text { otherwise }
\end{aligned}\right. \\
& \sum_{m, n} w_{m n}=1 \text {, } \\
& \mathbf{W} \equiv\left\{w_{m n}\right\} .
\end{aligned}
$$

Here $w_{m n}$ is simply the fraction of GCs in bin $m n, f_{m n}$ is the phase space density of GCs in bin $m n$, and $f(E, L)$ and $h_{m n}(E, L)$ are normalized so that their integrals over phase space within the survey limits are unity. 
In our calculation, the bin $m n$ is defined by

$$
\begin{aligned}
E_{m-1} & \leq E<E_{m} \\
\frac{n-1}{N_{L}} L_{c}\left(E_{m}\right) & \leq L<\frac{n}{N_{L}} L_{c}\left(E_{m}\right)
\end{aligned}
$$

where $E_{m-1}$ and $E_{m}$ are the lower and upper energy limits of the bin, and $L_{c}(E)$ is the maximum angular momentum at energy $E$, corresponding to a circular orbit. These bins cover all of the allowed region in $E-L$ space and may also cover some unallowed regions.

Each $h_{m n}(E, L)$ gives a normalized distribution in $\left(R, v_{z}\right)$ space,

$$
g_{m n}\left(R, v_{z}\right)=\int h_{m n}(E, L) d v_{x} d v_{y} d z
$$

Notice that $g_{m n}\left(R, v_{z}\right)$ depends on $\Phi$ (thus $\mathbf{X}$ ) but is independent of the DF specified by W. An algorithm for evaluating $g_{m n}\left(R, v_{z}\right)$ efficiently is shown in Appendix A. To account for errors in radial velocities, the distribution should be convolved with a one-dimensional Gaussian function as in equation (8). We use the convolved distribution hereafter and drop the prime symbol for simplicity.

Due to the linearity of equations (4) and (14), we have

$$
\begin{aligned}
g\left(R, v_{z}\right) & =\sum_{m, n} w_{m n} g_{m n}\left(R, v_{z}\right), \\
L H(\mathbf{X}, \mathbf{W}) & \propto \prod_{i} g\left(R_{i}, v_{z i}\right) \\
& =\prod_{i} \sum_{m, n} w_{m n} g_{m n}\left(R_{i}, v_{z i}\right) .
\end{aligned}
$$

Therefore, for a given set of observations $\left[R_{i}, v_{z i}\right]$, the likelihood is a function of $\mathbf{X}$ and $\mathbf{W}$ only. For a fixed potential specified by the parameters $\mathbf{X}$, we can calculate $g_{m n}\left(R_{i}, v_{z i}\right)$, then maximize $L H(\mathbf{X}, \mathbf{W})$ with respect to $\mathbf{W}$. We may then vary $\mathbf{X}$ to search for a global maximum $L H(\mathbf{X}, \mathbf{W})$.

An advantage of this method is that we need only ensure that all $w_{m n}$ are non-negative to guarantee that $f(E, L) \geq 0$. However, this method does not generally give smooth DFs. The derived DFs look like the sum of a set of $\delta$-functions ((Merritt 1993, see also figure 1c). This is a drawback, since to some extent the irregularities in the DFs are fitting the statistical fluctuations in the data rather than the signal. In other words it is an ill-posed problem to infer $g\left(R_{i}, v_{z i}\right)$ from a small set of data points. One way to approach this problem is to maximize a "penalized likelihood" instead, which can be defined using entropy maximization 
(Richstone \& Tremaine 1988) or regularization (Cox \& O'Sullivan 1990; Merritt 1993; Rix et al. 1997).

By adding an entropy term, we maximize the function

$$
\begin{aligned}
T & \equiv \ln L H+\alpha S, \\
S & \equiv-\int C(f) d^{3} \mathbf{x} d^{3} \mathbf{v},
\end{aligned}
$$

where $\alpha$ is a parameter chosen to control the degree of smoothness of the DF, and $C(f)$ is a convex function of $f$ such as $f \ln f$. Adding the entropy term tends to force the DF to be uniform in phase space (in the limit of large $\alpha$, the data become irrelevant and the maximum value of $T$ occurs for $f=$ const). This tendency is particularly undesirable in our case because there is a large phase volume at high energy, so that large $\alpha$ tends to place more GCs in a high energy state. We have done simulations to test this method and found that the derived DF is either too irregular for small $\alpha$ or has an excess high energy tail for large $\alpha$.

To implement regularization, we maximize the function

$$
Q(\mathbf{X}, \mathbf{W}) \equiv \ln L H-\lambda_{E} \Pi_{E}-\lambda_{L} \Pi_{L},
$$

where $\lambda_{E}$ and $\lambda_{L}$ are positive parameters that adjust the degree of smoothness of the DF and $\Pi_{E}$ and $\Pi_{L}$ are dimensionless positive-definite functions of the DF. There are several natural forms for $\Pi_{E}$, including

$$
\begin{array}{ll}
\Pi_{E}=\left\langle\left(\frac{\partial \ln f}{\partial\left(E / E_{c}\right)}\right)^{2}\right\rangle & \text { uniform form, } \\
\Pi_{E}=\left\langle\left|\frac{\partial^{2} \ln f}{\partial\left(E / E_{c}\right)^{2}}\right|\right\rangle \quad \text { exponential form, } \\
\Pi_{E}=\left\langle\left|\frac{\partial^{2} \ln f}{\partial \ln \left(E / E_{c}\right)^{2}}\right|\right\rangle \text { power-law form, }
\end{array}
$$

where $E_{c}$ is a normalizing constant. The names indicate what functions minimize $\Pi_{E}$; for example, the uniform form is zero if $f=$ const, the exponential form is zero if $f \propto \exp (-\beta E)$, where $\beta$ is a constant, etc. The angle brackets denote an average over area in $E-L / L_{c}(E)$ space; for example, if we use a fixed energy interval for all bins, the exponential form is proportional to

$$
\sum_{m, n}\left|\ln f_{(m+1) n}-2 \ln f_{m n}+\ln f_{(m-1) n}\right|,
$$

in case no $V_{m n}$ is zero. The term $\Pi_{L}$ smoothing the DF along the $L$ direction can be written similarly. 
Before we choose the form of $\Pi_{E}$ and $\Pi_{L}$, it is helpful to discuss the meaning of the regularization term. When equation (23) is compared to equation (1), we see that in these two cases we are maximizing

$$
e^{Q}=e^{-\lambda_{E} \Pi_{E}-\lambda_{L} \Pi_{L}} L H(\Phi, f)
$$

and

$$
P\left(\Phi, f \mid\left[R_{i}, v_{z i}\right]\right) \propto P\left(f \mid H_{f}\right) L H(\Phi, f),
$$

respectively. In the other words, maximizing $Q$ is equivalent to maximizing the posterior probability if the prior probability for $f$ is

$$
P\left(f \mid H_{f}\right) \equiv e^{-\lambda_{E} \Pi_{E}-\lambda_{L} \Pi_{L}} .
$$

By choosing $\lambda_{E}$ and $\Pi_{E}$, we are assuming some prior information $H_{f}$, i.e., a specific degree of smoothness and shape of the DF. Therefore, the smoothing terms assess the physical plausibility of the DF.

Generally, DFs of GCs are strongly non-uniform in the sense that they may change amplitude by a few orders of magnitude over the observable range. For example, given a logarithmic potential and a GC density profile

$$
\Phi=2 \sigma^{2} \ln r, \quad \nu(r) \propto r^{-3},
$$

the corresponding isotropic DF is

$$
f(E) \propto e^{-3 E / 2 \sigma^{2}} .
$$

Over the range $R_{0}<R<R_{1}$, the DF at zero velocity will vary by a factor of $\left(R_{1} / R_{0}\right)^{3}$, which is about 3000 , given that the ratio $R_{1} / R_{0}$ is $110 \mathrm{kpc} / 7 \mathrm{kpc} \sim 15$ in the case of M87 (see $\S 4$ ).

Given this large variation, neither entropy maximization as in equation (22) nor the uniform form of the regularization term in equation (24) is appropriate, because they tend to favor a uniform DF over the entire phase space. The exponential and power-law forms in equations (25) and (26) give more freedom and favor exponential or power-law forms for the $\mathrm{DF}$, which are more physically reasonable. We adopt the exponential form throughout this paper.

How should we choose $\lambda_{E}$ and $\lambda_{L}$ ? If these parameters are set too small, they do not smooth the DF at all. If they are set too large, the DF is forced to a specific functional 
form that may not be demanded by the data. Notice that in contrast to many inverse problems (Press et al. 2003) the likelihood is only known to within a multiplicative constant, so the goodness of fit can only be assessed in a relative sense. We choose the parameters as follows. First, we choose $\lambda_{E}$ by setting $N_{L}=1$ (isotropic DF): in the limit of zero and infinitely large smoothing parameter $\lambda_{E}$, we may get maximum panelized likelihood $Q_{1}$ and $Q_{2}$, respectively. We choose smoothing parameter $\lambda_{E}$ so that the maximum panelized likelihood is $\left(Q_{1}+Q_{2}\right) / 2$. Second, we allow anisotropy and fix $\lambda_{E}$ at the value obtained in the isotropic case, then choose $\lambda_{L}$ similarly. We call these "natural" smoothing parameters. This subjective method of choosing smoothing parameters is not justified by any formal argument. However, it does reflect a trade-off between the prior and information included in observed data.

For convenience, we define

$$
Q_{\max }(\mathbf{X}) \equiv \max _{\mathbf{W}} Q(\mathbf{W}, \mathbf{X})
$$

which is $Q$ maximized with respect to $\mathbf{W}$. The function $Q(\mathbf{W}, \mathbf{X})$ usually has a number of local maxima in $\mathbf{W}$ at fixed $\mathbf{X}$. We attempt to find the global maximum in this landscape using simulated annealing and the downhill simplex method, as discussed in Press et al. (2003). The method does not guarantee that we have found the global maximum, so we try several different initial values of $\mathbf{W}$ and choose the largest maximum found from any of these initial conditions. The optimization process turns out to consume most of the computational resources required by this method.

We may also assume some prior information $H_{X}$ for the potential parameters, which gives a prior probability $P\left(\mathbf{X} \mid H_{X}\right)$. For example, in the power-law potential (eq. 9), it is reasonable to assume a uniform prior in $\left(\ln \rho_{0}, \alpha\right)$, thus we have

$$
P\left(\mathbf{X} \mid H_{X}\right)=\rho_{0}^{-1}
$$

Then the probability distribution of the potential parameters $\mathbf{X}$ is

$$
P(\mathbf{X}) \propto P\left(\mathbf{X} \mid H_{X}\right) Q_{\max }(\mathbf{X})
$$

We may also incorporate the observed surface number density profile $\Sigma_{0}\left(R_{j}\right)$ of the GCs and its error $\sigma_{\Sigma}\left(R_{j}\right)$ as an additional constraint in our calculation since this may be available for many more GCs, over a larger survey area than have measured velocities. We maximize the quantity

$$
Q^{\prime} \equiv \ln L H-\frac{\chi^{2}}{2}-\lambda_{E} \Pi_{E}-\lambda_{L} \Pi_{L}
$$




$$
\begin{aligned}
\chi^{2} & =\sum_{j} \frac{\left(\Sigma\left(R_{j}\right)-\Sigma_{0}\left(R_{j}\right)\right)^{2}}{\sigma_{\Sigma}\left(R_{j}\right)^{2}} \\
\Sigma(R) & \propto \int g\left(R, v_{z}\right) d v_{z} \\
& =\sum_{m, n} w_{m n} \int g_{m n}\left(R, v_{z}\right) d v_{z}
\end{aligned}
$$

where both $\Sigma(R)$ and $\Sigma_{0}(R)$ should be normalized so that $2 \pi \int R d R \Sigma(R)$ and $2 \pi \int R d R \Sigma_{0}(R)$ are unity within the survey limit of GC number counting.

\section{Harmonic oscillator potential}

As a simple test case, we first study a GC system in a harmonic oscillator potential. The potential is

$$
\Phi(r)=\frac{1}{2} \Omega^{2} r^{2}, \quad r \equiv \sqrt{R^{2}+z^{2}}
$$

The simulated data are generated using $\Omega=1$ and the DF

$$
f(E)=C\left(e^{-E / 10}+\frac{1}{20} e^{-(E-100)^{2} / 900}\right)
$$

where $E \equiv \Phi(r)+\frac{1}{2}\left(v_{R}^{2}+v_{z}^{2}\right), v_{R}$ is the transverse velocity normal to the line of sight, $v_{z}$ is the velocity in the line of sight, and $C$ is a constant chosen so that the DF is normalized to unity. The combination of the exponential and Gaussian function in the DF is chosen so that the energy distribution of GCs is bimodal, to challenge our algorithm.

Using this DF and potential, we generate 160 pairs of observables $\left[R_{i}, v_{z i}\right]$. We then try to derive $\Omega$ from the data by maximizing $Q_{\max }(\Omega)$ (eq. 33) with respect to $\Omega$.

\subsection{Isotropic DF}

We first assume that the DF is isotropic. To carry out this optimization we use 40 bins in energy space, i.e., $N_{E}=40$ and $N_{L}=1$. We use the exponential form of the regularization term (eq. 25). Figure 1a shows $Q_{\max }(\Omega)$ for smoothing parameters $\lambda_{E}=0,0.05$ and 0.5 , where $\lambda_{E}=0.05$ is the natural smoothing parameter as defined in $\S 2$. The quantity $Q_{\max }(\Omega)$

decreases with $\lambda_{E}$ for two reasons: first, the regularization term $\lambda_{E} \Pi_{E}$ tends to be larger for larger $\lambda_{E}$; second, larger $\lambda_{E}$ means less freedom to adjust the shape of the DF to fit the data, 
which leads to a smaller likelihood $L H(\Omega, \mathbf{W})$. For all three values of $\lambda_{E}$, the peaks are close to the input value $\Omega=1.0$, so the method can recover the correct potential parameters with or without regularization.

Figures $1 \mathrm{~b}$ and $1 \mathrm{c}$ show the derived DFs and the energy distribution $\mathbf{W}$, respectively. The DFs and $\mathbf{W}$ for different values of $\lambda_{E}$ are shifted by an arbitrary amount for clarity. The solid lines are the input DF and $\mathbf{W}$ given by equation (40). Without regularization $\left(\lambda_{E}=0\right)$, the derived DF and $\mathbf{W}$ are irregular (dotted lines), although they do hint at the bimodal character of the input DF.

The DF is over-smoothed for $\lambda_{E}=0.5$ (dash-dotted lines): the DF is biased by the smoothing and the bimodal energy distribution is not recovered.

The dashed lines show that with the natural smoothing parameter $\lambda_{E}=0.05$, regularization successfully smooths the DF to a form close to the input DF. The bimodal energy distribution is recovered well. The differences between the derived and input DFs are mostly due to statistical noise in the simulated data, which is greatly reduced in a larger simulation run with 2000 data points.

The confidence level of $\Omega$ can be determined in two ways: (i) identify the interval of $\Omega$ that gives $\ln Q_{\max }(\Omega) \geq \max _{\Omega} \ln \left(Q_{\max }(\Omega)\right)-\frac{1}{2}$. (ii) bootstrap estimation. For $\lambda_{E}=0.05$, the two methods give $\Omega=0.94 \pm 0.07$ and $\Omega=0.95 \pm 0.08$, respectively. Both methods have similar accuracy, but the first method needs far fewer calculations.

We may compare this estimate of $\Omega$ to an estimate obtained by applying the virial theorem to the same data. The virial theorem states that

$$
\begin{gathered}
\left\langle v^{2}\right\rangle=\Omega^{2}\left\langle r^{2}\right\rangle, \\
\Omega=\sqrt{\frac{2\left\langle v_{z}^{2}\right\rangle}{\left\langle R^{2}\right\rangle} .}
\end{gathered}
$$

Applying bootstrap estimation, we get $\Omega=0.95 \pm 0.07$. The virial theorem estimate is much simpler than the method described here, but cannot be generalized to potentials depending on several parameters and does not yield an estimate of the DF.

\subsection{Anisotropic DF}

We now re-analyze the same data, without the assumption that the DF is isotropic. We are trying to find out whether the data can constrain the isotropy of the DF and whether allowing a more general DF degrades the accuracy of the determination of $\Omega$. 
To carry out the optimization, we divide $E-L / L_{c}(E)$ space into $20 \times 5$ bins. Figure 2a shows $Q_{\max }(\Omega)$ for $\lambda_{E}, \lambda_{L}=0.00,0.05,0.50$. The middle values, $\lambda_{E}, \lambda_{L}=0.05$, are the natural smoothing parameters as defined in $\S 2$. Comparing Figures $1 \mathrm{a}$ and $2 \mathrm{a}$, we see that allowing anisotropy increases $Q_{\max }(\Omega)$ for $\lambda_{E}, \lambda_{L}=0.00$ and 0.05 but not for $\lambda_{E}, \lambda_{L}=0.50$. The reason is that for modest smoothing, anisotropy allows more freedom to adjust the DF to fit the data, thus giving a larger likelihood, while for large smoothing, the DF becomes flat in the $L$ direction, so the likelihood is almost the same as with the isotropic assumption. We find $\Omega=0.94 \pm 0.08$ for $\lambda_{E}, \lambda_{L}=0.05$. Thus we still get a reliable estimation for $\Omega$, even without assuming isotropy.

Figure $2 \mathrm{~b}$ shows the energy distribution of the GCs, described by

$$
\mathbf{U} \equiv\left\{\sum_{n} w_{m n}\right\}
$$

i.e., the sum of the weights over all angular-momentum bins at a given energy. It fits the input distribution (dotted line) reasonably well for $\lambda_{E}, \lambda_{L}=0.05$. Notice that $\mathbf{U}=\mathbf{W}$ if $N_{L}=1$.

Figure 3a shows the quantity

$$
I(E, L) \equiv \frac{f(E, L)}{\bar{f}(E)} \text { or } I_{m n} \equiv \frac{f_{m n}}{\bar{f}(E)}
$$

for $\lambda_{E}, \lambda_{L}=0.05$, where $\bar{f}(E)$ is the DF averaged over phase space at energy $E$,

$$
\begin{aligned}
\bar{f}(E) & \equiv \frac{\int_{E}^{E+\delta E} f(E, L) d^{3} \mathbf{x} d^{3} \mathbf{v}}{\int_{E}^{E+\delta E} d^{3} \mathbf{x} d^{3} \mathbf{v}} \\
& =\frac{\sum_{n} w_{m n}}{\sum_{n} V_{m n}}
\end{aligned}
$$

Figure $3 \mathrm{~b}$ shows the weighted indicator of anisotropy

$$
J\left(L / L_{c}\right) \equiv J_{n} \equiv \sum_{m} I_{m n} U_{m}
$$

which is a constant for an isotropic DF. The ratio $I(E, L)$ varies from 0.2 to 2.4 while $J\left(L / L_{c}\right)$ does not differ significantly from a constant for the chosen smoothing parameters $\lambda_{E}, \lambda_{L}=0.05$. We cannot claim that the analysis has recovered the isotropy of the original DF although the flatness of $J\left(L / L_{c}\right)$ for $\lambda_{E}, \lambda_{L}=0.05$ does hint at an isotropic DF.

Our simulations suggest that we can recover the dependence of the DF on energy $E$ reasonably well with a sample of this size but we can not recover the dependence of DF on 
angular momentum $L$. This is because the observable distribution $g\left(R, v_{z}\right)$ depends more strongly on how stars are distributed in different $E$ levels than on how GCs are distributed in different $L$ levels.

\section{Mass distribution of M87}

\subsection{A uniform dataset}

Surveys of GC positions and radial velocities for M87 are described in Section 1. The largest survey of GC radial velocities is given by Côté et al. (2001), who list 278 GCs. However, they are drawn from a variety of sources. To construct a uniform sample, we use only the data in Cohen and Ryzhov (1997): these are drawn from a survey by Strom et al. (1981), which was complete only in the radius range $90^{\prime \prime}-405^{\prime \prime}$. So we have the survey limits $\left(R_{s 0}, R_{s 1}\right)=(7,32) \mathrm{kpc}$ (see Figure 4$)$. Restricting our sample to this range, we have 161 GCs, which are shown in Figure 5. The errors in radial velocities given by Cohen and Ryzhov (1997) range between $50-100 \mathrm{~km} \mathrm{~s}^{-1}$; we set the errors equal to $75 \mathrm{~km} \mathrm{~s}^{-1}$ for all GCs.

\subsection{Analysis}

We have described our method in $\S 2$. In constructing our models we must choose a maximum energy $E_{N_{E}}$ (eq. 18) for the cluster population, or equivalently, a largest apocenter $r_{m}$ such that $\Phi\left(r_{m}\right)=E_{N_{E}}$. We choose $r_{m}=300 \mathrm{kpc}, 9$ times larger than the outer survey radius $R_{s 1}=32 \mathrm{kpc}$. If $r_{m}$ is too small, we bias our results by excluding high-energy GCs that may be present within the survey radii. If $r_{m}$ is too large, the numerical work will be dominated by GCs that spend only a small fraction of their orbits within the survey radii. For comparison, Harris (1986) observed GCs out to projected radius $R=110 \mathrm{kpc}$.

We may also incorporate the surface number density profile $\Sigma_{0}\left(R_{j}\right)$, which is given with estimated error $\sigma_{\Sigma}\left(R_{j}\right)$ in 25 bins from 7 to $110 \mathrm{kpc}$ by Harris (1986) and McLaughlin (1999a), as an additional constraint in our calculation, as described in $\S 2$. Both $\Sigma(R)$ and $\Sigma_{0}(R)$ should be normalized so that $2 \pi \int R d R \Sigma(R)$ and $2 \pi \int R d R \Sigma_{0}(R)$ are unity within the radius range $7-110 \mathrm{kpc}$. Notice that we do double count some of the GCs in $7-32 \mathrm{kpc}$ since they appear in both the kinematic data $\left[R_{i}, v_{z i}\right]$ and in the surface density distribution $\Sigma_{0}\left(R_{j}\right)$. However, this does not affect our results in a noticeable way because there are over 7000 GCs used in the calculation of the surface density distribution. 


\subsection{Results}

\subsubsection{Isotropic power-law models}

Initially we assume that the DF is isotropic $\left(N_{E}=80, N_{L}=1\right)$ and the mass density profile of the galaxy is a power law (eq. 9). We set the arbitrary radius $r_{0}$ in equation (9) to be $19 \mathrm{kpc}$, near the median radius of our samples to minimize the covariance of $\rho_{0}$ and $\alpha$ in their bivariate probability distribution.

In this case, $\mathbf{X}=\left\{\rho_{0}, \alpha\right\}$. Figure 6a shows the contour plot for $Q_{\max }(\mathbf{X})$. The natural smoothing parameter is $\lambda_{E}=0.0005$. The plus sign is the best-fit model which gives the maximum $Q_{\max }(\mathbf{X})$. The contours are $n-\sigma$ levels from the peak, i.e., with $e^{-n^{2} / 2}$ of the maximum likelihood. Figure $6 \mathrm{~b}$ shows that the derived number density profile $\Sigma(R)$ for the best-fit model (dotted line) is roughly consistent with the observed profile $\Sigma_{0}(R)$ (solid line) out to $100 \mathrm{kpc}$, even though we have not fit the number density profile $\Sigma_{0}(R)$ and thus only use data within $R_{s 1}=32 \mathrm{kpc}$ (i.e., we maximize $Q$ of eq. 33 rather than $Q^{\prime}$ of eq. 36). So to some extent, our methods explore the potential beyond the maximum survey radius $R_{s 1}$.

Figure 7 shows the results if we fit for the surface number density profile as well, by maximizing $Q^{\prime}(\mathbf{X}, \mathbf{W})$ with respect to $\mathbf{W}$. The best-fit parameters $\mathbf{X}$ change by $\sim 10 \%$ or less than $1-\sigma$, and the uncertainties shrink by $\sim 60 \%$. This is expected since the number density profile contains extra information.

Figure 8 shows the derived energy distribution of the GCs. Zero energy has been set as the potential energy at $r=7 \mathrm{kpc}$. The dash-dotted line (bottom) and dotted line (middle) are the energy distributions of GCs within projected radius $R=7-32 \mathrm{kpc}$, which are calculated from the DF associated with the best-fit model in 6a and 7a, respectively. The upper dashed line is the energy distribution of all GCs with $R \geq 7 \mathrm{kpc}$, calculated from the DF associated with the best-fit model in 7a. These lines suggest that there may be kinematically distinct groups of GCs in M87.

\subsubsection{Anisotropic power-law models}

Figures $9 \mathrm{a}$ and $9 \mathrm{~b}$ show the result of fitting models with power-law potentials and anisotropic DFs $\left(N_{E}=40, N_{L}=5\right)$ to both the kinematic data and the observed surface number density, using $\lambda_{E}, \lambda_{L}=0$ and the natural smoothing parameters $\left(\lambda_{E}, \lambda_{L}\right)=$ $(0.0025,0.60)$, respectively. The contours in Figure 9a (without regularization) and Figure $9 \mathrm{~b}$ (with natural smoothing parameters) are very similar, suggesting that the estimate of potential parameters of M87 is rather insensitive to smoothing parameters. The contour 
in Figure $9 \mathrm{~b}$ is more irregular and the uncertainties are larger than in Figure $7 \mathrm{a}$, which is expected since anisotropy gives more freedom to adjust the DF to fit the data; however, the best-fit potential parameters $(\rho, \alpha)=\left(1.9 \times 10^{7} \mathrm{M}_{\odot} / \mathrm{kpc}^{3}, 1.9\right)$ (plus symbol) does not change from that for isotropic models. A secondary peak is marked by the asterisk symbol, with $Q^{\prime}$ smaller by 0.2 . The derived surface number density from the best-fit model in Figure $9 \mathrm{c}$ has a stronger tail than that derived under the assumption of isotropy (see Figure 7b). Figure $9 \mathrm{~d}$ shows the energy distributions of GCs within projected radius $R=7-32 \mathrm{kpc}$ (solid line) and with $R \geq 7 \mathrm{kpc}$ (dotted line), which are also composed of a few peaks. The peak at $E \simeq 135 \times\left(100 \mathrm{~km} \mathrm{~s}^{-1}\right)^{2}$ accounts for the strong tail in the derived surface number density profile beyond $100 \mathrm{kpc}$.

These results are based on the estimate of background stars $5.8 \pm 0.3$ stars $\operatorname{arcmin}^{-2}$ (Harris 1986). We also investigate the case of 6.1 stars $\operatorname{arcmin}^{-2}$. We get almost the same likelihood contours; however, the amplitude of the high-energy tail is reduced by a factor of 2 .

Figure 10 shows the indicators of anisotropy $I(E, L)$ and $J\left(L / L_{c}\right)$. The results suggest that the GCs prefer circular orbits (high angular momentum) for the best-fit model but radial orbits (low angular momentum) at the secondary peak. To reliably determine the anisotropy of the GCs, we need to obtain more data to give a tighter constraint on mass distribution and develop a more robust formalism of choosing smoothing parameters in the future.

To estimate the probability distribution of parameters derived from the potential, we lay down points in Figure $9 \mathrm{~b}$ with a uniform probability distribution in $\left(\ln \rho_{0}, \alpha\right)$, then reject or save each point according to $Q^{\prime}(\mathbf{X}, \mathbf{W})$ at that point. From the saved points, we may estimate the distribution of $M(r)$, the mass enclosed within radius $r$, and the exponent $\alpha$. Figure 11a shows the probability distribution of $M(r=32 \mathrm{kpc})$, which is derived from $4 \times 10^{4}$ points and has been normalized to its maximum. Figure 11 b shows the probability distribution of the density slope $\alpha$. The data provide a rather tight constraint on $\alpha$, which is $1.6 \pm 0.4$ ( $1-\sigma$ error $)$.

If we stack curves like that in Figure 11a, we get the mass distribution for all radii, which is shown in Figure 12a. The solid curves mark the mean and 1,2,3- $\sigma$ errors. The solid line and error bars in Figure 12b show the best estimate of $M(r)$ and its standard deviation, which gives $M(32 \mathrm{kpc})=2.3 \pm 0.7 \times 10^{12} M_{\odot}$. Notice that the relative error is the smallest around $32 \mathrm{kpc}$, which is reasonable since it is the maximum survey radius. For comparison, isotropic models in $\S 4.3 .1$ give $M(32 \mathrm{kpc})=2.6 \pm 0.3 \times 10^{12} M_{\odot}$ and $\alpha=1.8 \pm 0.2$, which differ by less than the error bars. 
Also shown in Figure 12b are previously published models. The data for X-ray observations (Nulsen \& Böhringer 1995) were obtained by assuming a distance of 20 Mpc to M87. The long dashed lines show their derived lower and higher limits scaled by a factor of 0.815 since we assume a distance of $16.3 \mathrm{Mpc}$. Our mass estimation is consistent with the X-ray observations in the range where they overlap. The estimation by McLaughlin (1999b) is based on X-ray observations and an assumption of a NFW dark matter halo of the Virgo cluster. The estimations by Merritt (1993) and Cohen and Ryzhov (1997) are based on the assumption of an isotropic DF.

Figure 13 shows the derived velocity dispersion profile (dotted line)

$$
\sigma_{z}(R)=\left\langle v_{z}^{2}\right\rangle^{1 / 2}=\left(\frac{\int v_{z}^{2} g\left(R, v_{z}\right) d v_{z}}{\int g\left(R, v_{z}\right) d v_{z}}\right)^{1 / 2}
$$

which is consistent with observed velocity dispersion profile (dashed line with error bars).

\subsubsection{Other anisotropic models}

We also investigate models with two other potentials, (i) a NFW profile (eq. 10), (ii) the potential generated by the stars, assuming constant mass-to-light ratio. The dotted line in Figure 12b shows the estimate for $M(r)$ for NFW models, which is almost the same as that for power-law models beyond $20 \mathrm{kpc}$. The errors are comparable to those for power-law models. The best-fit constant mass-to-light ratio model has $M / L=125 \pm 10 \mathrm{M}_{\odot} / \mathrm{L}_{\odot}$. The maximum $Q^{\prime}$ for the best-fit powerlaw and NFW models differ by less than 1- $\sigma$ while the maximum for constant $M / L$ is smaller by 7 , i.e., about $4-\sigma$ worse than for the NFW or power-law models.

We further check our results by simulated data. We choose an isotropic DF

$$
f(E)=e^{-(E-320)^{2} / 100}+0.04 e^{-(E-385)^{2} / 100}
$$

in a power-law potential with $r_{0}=19$ and $\left(4 \pi G \rho_{0}, \alpha\right)=(10,1.9)$. The DF is chosen so that the energy distribution is bimodal. We generate 160 kinematic data points within 7-32 and surface number density profile within 7-110 with 7000 GCs. We get good mass estimations even when the smoothing parameters are large enough to force the DF to be exponential form. For the chosen appropriate smoothing parameters, we recover the bimodal energy distribution successfully. 


\section{Discussion}

We have investigated how to determine the mass distribution of a spherical stellar system from test particle kinematics. We use a non-parametric form for the DF, which includes the isotropic DF as a spherical case, and parametrized forms for the potential, we find the bestfit potential and DF using a penalized maximum likelihood method. Our method allows for selection effects, observational and statistical errors, and anisotropy in the DF. We find that the potential parameters are determined more securely than the energy distribution, which is in turn more accurate than the angular-momentum distribution. Our simulations show that mass distributions depending on two parameters can be derived from a small number $(\leq 200)$ of data pairs $\left[R_{i}, v_{z i}\right]$ rather accurately, Plausible estimates of the energy distribution of the test particles can also be recovered; however, we can not tell whether the DFs are isotropic or not from such a small dataset.

We have applied our method to a sample of 161 GCs between $7 \mathrm{kpc}$ and $32 \mathrm{kpc}$ in M87. Under the assumption that the system is spherical and the DF is isotropic, we infer that the mass of M87 within $32 \mathrm{kpc}$ is $2.6 \pm 0.3 \times 10^{12} \mathrm{M}_{\odot}$. The power-law index for the density profile, $\rho \propto r^{-\alpha}$ is $\alpha=1.8 \pm 0.2$. If we allow an anisotropic DF, the mass M 87 within $32 \mathrm{kpc}$ is $2.3 \pm 0.7 \times 10^{12} \mathrm{M}_{\odot}$ and the power-law index is $1.6 \pm 0.4$, within 1 -standard deviation of the results for an isotropic DF. Our method yields more reliable and accurate results than earlier attempts to measure the mass of M87 from GC kinematics (Merritt \& Tremblay 1993; Cohen and Ryzhov 1997; Romanowsky \& Kochanek 2001).

We find that our mass estimates are insensitive to the smoothing parameters. Smoothing has stronger effects on the DF, which is determined much less reliably. The energy distribution has multiple peaks for the chosen smoothing parameters, perhaps suggesting a few kinematically distinct groups of GCs in M87. The derived anisotropy of GCs is strongly correlated with the mass model, thus cannot be determined at this stage. With more data, it may be possible to measure the anisotropy of the velocity distribution and test models of GC evolution predicting that GCs on less-eccentric orbits are more likely to survive (e.g. Gnedin \& Ostriker 1997). A more statistically robust method of choosing smoothing parameters, more kinematic data and a more accurate surface number density, especially beyond 110 kpc are the most important theoretical and observational advances needed to determine the mass distribution in M87 more accurately by this method.

We thank Judy Cohen for providing data in Figures 4 and 5 in tabular form, Michael Strauss, David Spergel, James Gunn and Robert Lupton for valuable discussions. This research was supported in part by NASA grant NNG04GL47G and used computational facilities supported by NSF grant AST-0216105. 


\section{A. Calculation of $g_{m n}\left(R, v_{z}\right)$}

We divide $E-L$ space into $N_{E} \times N_{L}$ rectangular bins. Assume that the bin $m n$ is defined by

$$
\begin{aligned}
E & \in\left[E_{m-1}, E_{m}\right], \\
L & \in\left[L_{n-1}, L_{n}\right] .
\end{aligned}
$$

We wish to determine the normalized distribution $g_{m n}\left(R, v_{z}\right)$ in projected radius and radial velocity corresponding to a uniform phase-space density in bin $m n$ (eq. 19). For an anisotropic DF, this requires a three-dimensional integration over $v_{x}, v_{y}$ and $z$, which is computationally expensive. We may achieve considerate speedup by integrating analytically

over $v_{y}$ (recall from eq. 5 that we assume that each GC lies on the $x$-axis). We have two constraints on $v_{y}$ from inequalities (A1). Based on equation (5), we have

$$
\begin{aligned}
v_{y}^{2} & \in[A, B] \\
& \equiv\left[2\left(E_{m-1}-\Phi(r)\right)-\left(v_{x}^{2}+v_{z}^{2}\right), 2\left(E_{m}-\Phi(r)\right)-\left(v_{x}^{2}+v_{z}^{2}\right)\right], \\
v_{y}^{2} & \in[C, D] \\
& \equiv\left[\frac{L_{n-1}^{2}-\left(v_{z} R-v_{x} z\right)^{2}}{\left(R^{2}+z^{2}\right)}, \frac{L_{n}^{2}-\left(v_{z} R-v_{x} z\right)^{2}}{\left(R^{2}+z^{2}\right)}\right] .
\end{aligned}
$$

Then we may set

$$
I \equiv \max (\min (B, D), 0) \quad J \equiv \max (\max (A, C), 0),
$$

so that we have

$$
\int d v_{y}=\max ((\sqrt{J}-\sqrt{I}), 0)
$$

This algorithm speeds up the integration by a factor of 20 or so.

\section{REFERENCES}

Côté, P. et al., 2001, ApJ, 559, 828

Cohen, J. G., 2000, AJ, 119, 162

Cohen, J. G., \& Ryzhov, A., 1997, ApJ, 486, 230

Cohen, J. G., Blakeslee, J. P., \& Ryzhov, A., 1998, ApJ, 496, 808 
Cox D. D., \& O’Sullivan, F., 1990, the Annals of Statistics, 18, 1676

Dirsch, B. et al., 2004, AJ, 127, 2114

Douglas, N. G. et al., 2002, PASP, 114, 1234

Gnedin, O. Y. \& Ostriker, J. P., 1997, ApJ, 474, 223

Harris W. E., 1986, AJ, 91, 4

Kendall, M., Stuart, A. \& Ord, J. K., 1987, Kendall's advanced theory of statistics (New York: Oxford Univ. Press)

Kissler-Patig, M., \& Gebhardt, K., 1998, AJ, 116, 2237

McLaughlin, D E., 1999a, AJ, 117, 2398

McLaughlin, D E., 1999b, ApJ, 512, L9

Merritt, D., 1993, ApJ, 413, 79

Merritt, D., \& Saha, P., 1993, ApJ, 409, 75

Merritt, D., \& Tremblay, B., 1993, AJ, 106, 2229

Mould, J. R., Oke, J. B., De Zeeuw, P. T., \& Nemec, J. M. 1990, AJ, 99, 1823

Navarro, J. F., Frenk, C. S., \& White, Simon D. M., 1997, ApJ, 490, 493

Nulsen, P. E. J., Böhringer, H., 1995, MNRAS, 274, 1093

Press, W. H., Flannery, B. P., Teukolsky, S. A., Vetterling, W. T., 2003, Numerical Recipes in Fortran (Cambridge: Cambridge Univ. Press)

Richstone, D. O., \& Tremaine, S., 1988, ApJ, 327, 82

Rix, H. et al., 1997, ApJ, 702, 719

Romanowsky, A. J., \& Kochanek, C. S. , 2001, ApJ, 553, 722

Strom, S. E., Forte, J., Harris, W., Strom, K. M., Wells, D., \& Smith, M. 1981, ApJ, 245, 416

Zeilinger, W. W., Møller, P., \& Stiavelli, M. 1993, MNRAS, 261, 175 

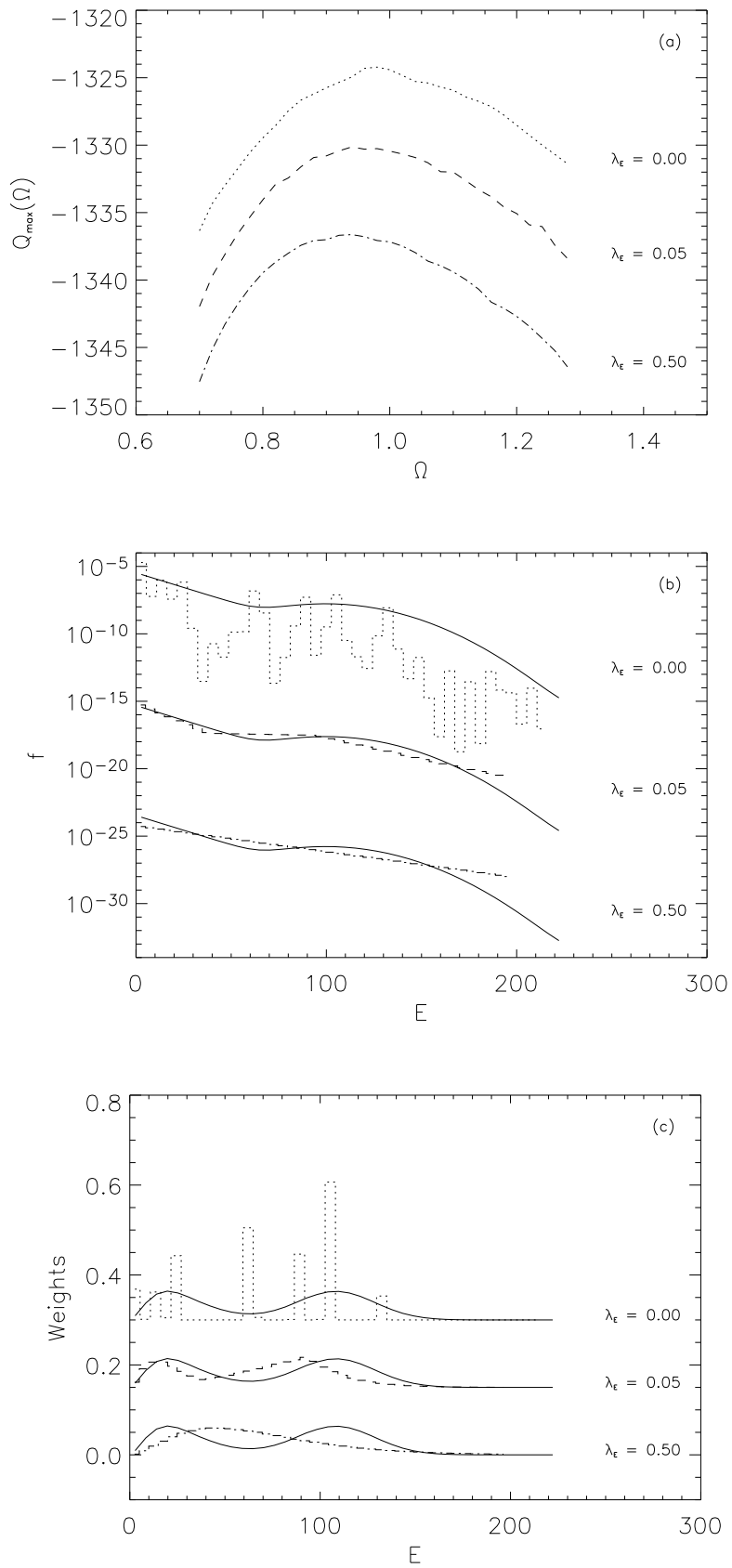

Fig. 1. - Tests of the recovery of the frequency $\Omega$ and the DF, using 160 data points in a harmonic potential (eq. 40). The DF is assumed to be isotropic (eq. 40) and equation (25) is used for regularization. (a) $Q_{\max }(\Omega)$ (eq. 33), a measure of the log of the regularized likelihood. The smoothness coefficient $\lambda_{E}=0.00,0.05,0.50$. The natural smoothing parameter is $\lambda_{E}=0.05$. The input potential parameter $\Omega=1$ is recovered well with all three values of $\lambda_{E}$. (b) The histograms show derived DFs and the solid lines show the actual DF on a logarithmic scale. For $\lambda_{E}=0.00$, the derived DF is not smooth at all; the DF is recovered pretty well for $\lambda_{E}=0.05$; and the derived DF is clearly over-smoothed for $\lambda_{E}=0.50$. (c) The histograms show the derived energy distribution $\mathbf{W}$ and the solid lines show the values calculated from the assumed DF in equation (40), on a linear scale. In (b) and (c), we shift 

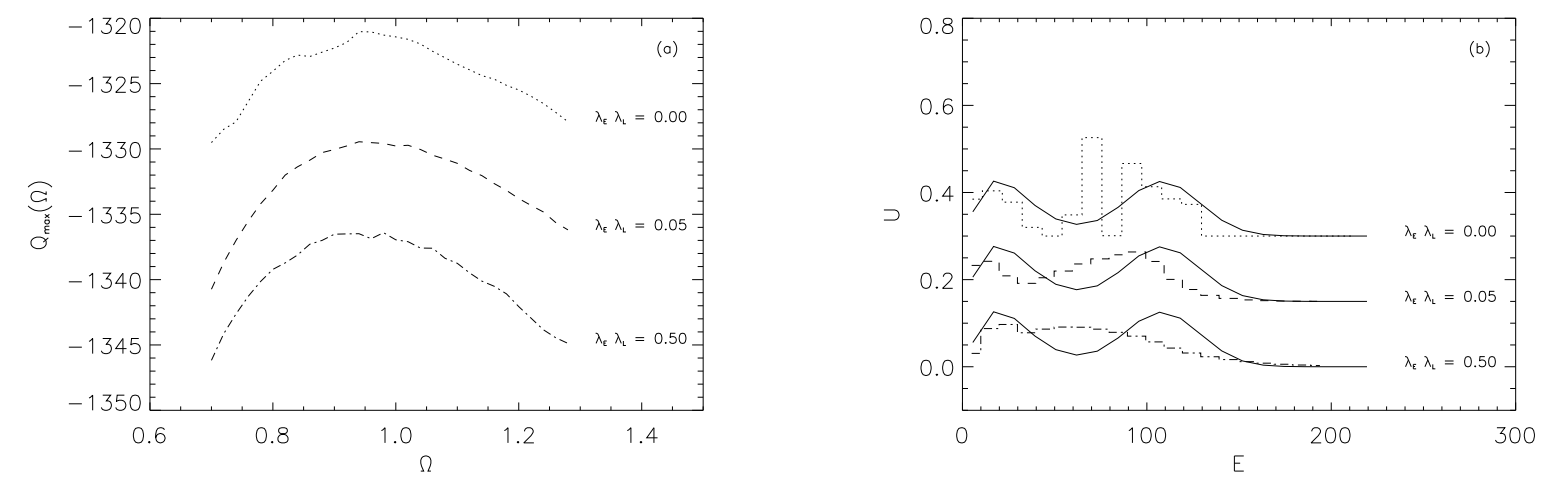

Fig. 2.- A test of the recovery of the potential and the DF, without assuming isotropy. The data consist of 160 points chosen from the DF in equation (40). The smoothness coefficients $\lambda_{E}, \lambda_{L}=0,0.05,0.5$. (a) $Q_{\max }(\Omega)$; the maximum likelihood is consistent with the input value $\Omega=1$. (b) The histogram shows the derived energy distribution $\mathbf{U} \equiv\left\{\sum_{n} w_{m n}\right\}$, i.e., the total fraction of GCs in each energy interval shifted vertically by arbitrary amounts. The dotted line shows the values calculated from the input DF. This test suggests that we can recover the energy distribution from a small $(\sim 160)$ data set. 

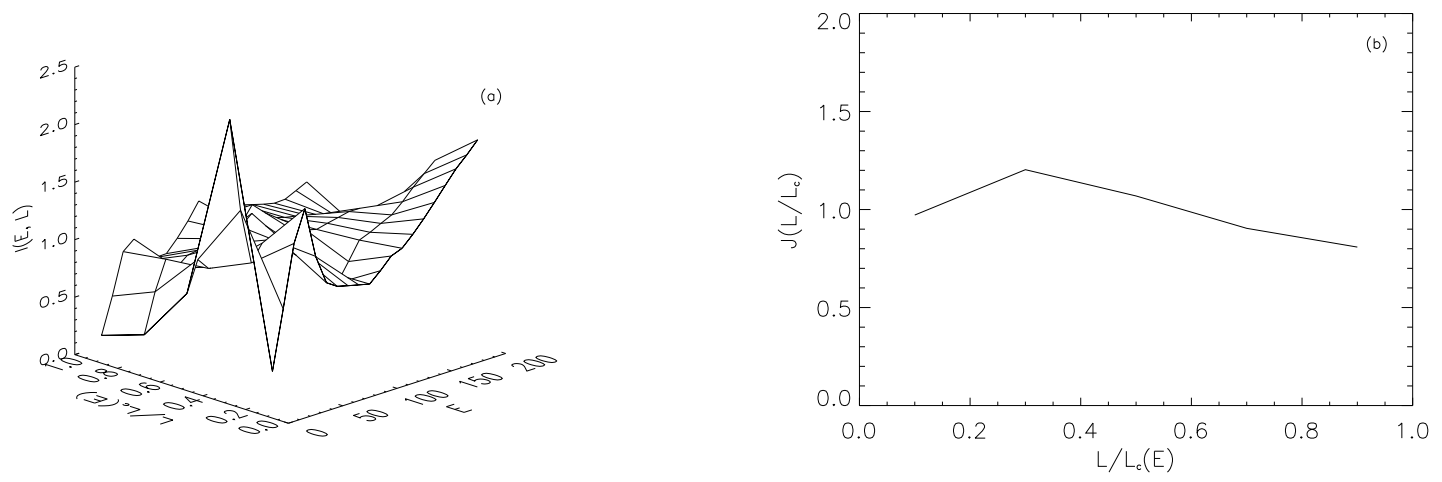

Fig. 3.- Indicators of anisotropy for $\lambda_{E}, \lambda_{L}=0.05$. (a) $I(E, L)$, the ratio of the DF to the isotropized DF (eq. 43). The irregular behavior of this indicator shows that we cannot accurately recover the anisotropy of the DF with this data set. (b) $J\left(L / L_{c}\right)$ (eq. 45 ), which appears consistent with an isotropic DF. However, this consistency is difficult to justify statistically. 


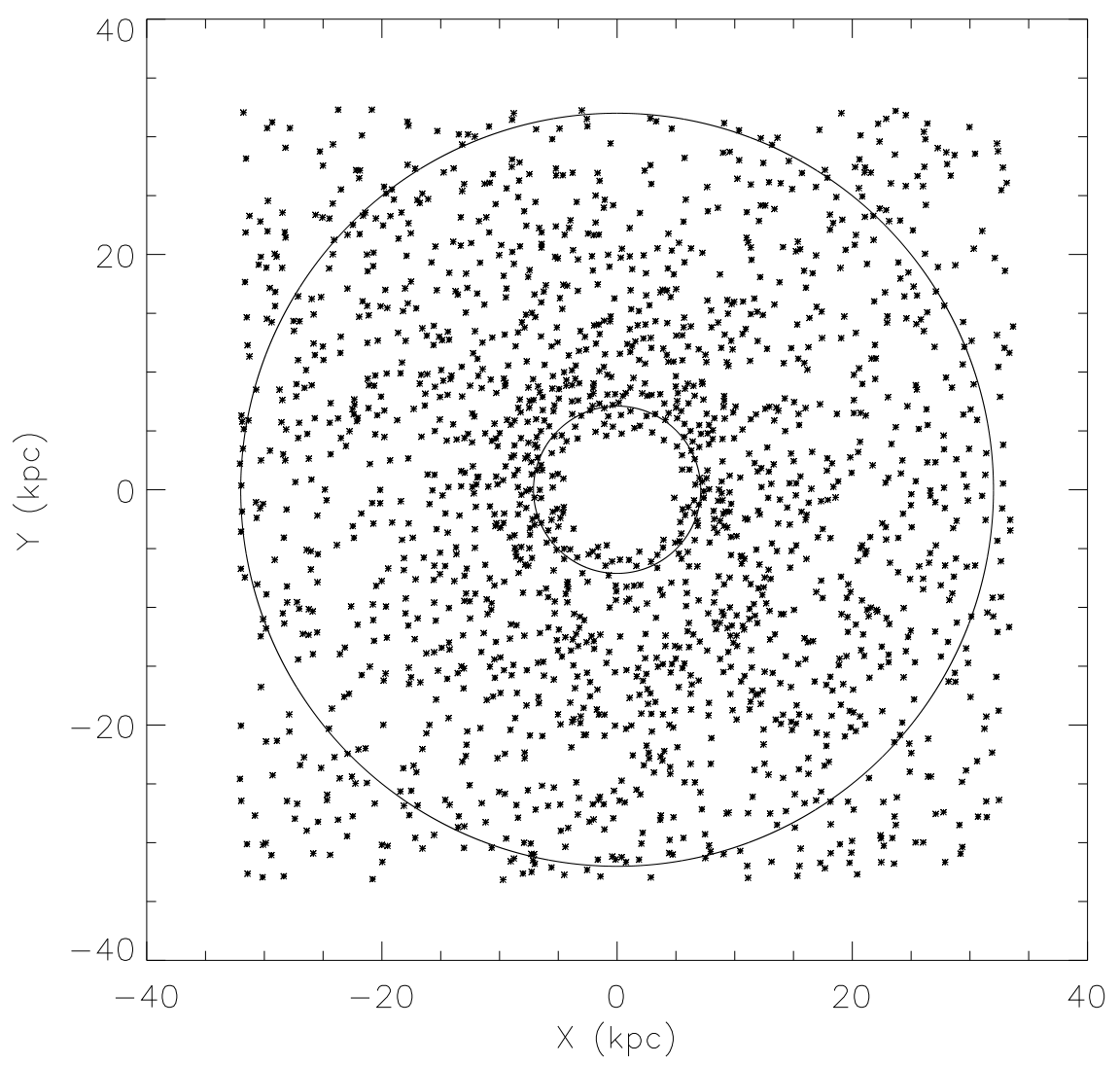

Fig. 4. - Spatial distribution of GC candidates in Cohen and Ryzhov (1997), which are taken from the photometric survey by Strom et al. (1981). To eliminate selection effects, we only use data between the two circles, which are $90^{\prime \prime}-405^{\prime \prime}$ or $7-32 \mathrm{kpc}$ from the center, where the Strom et al. survey was complete. 


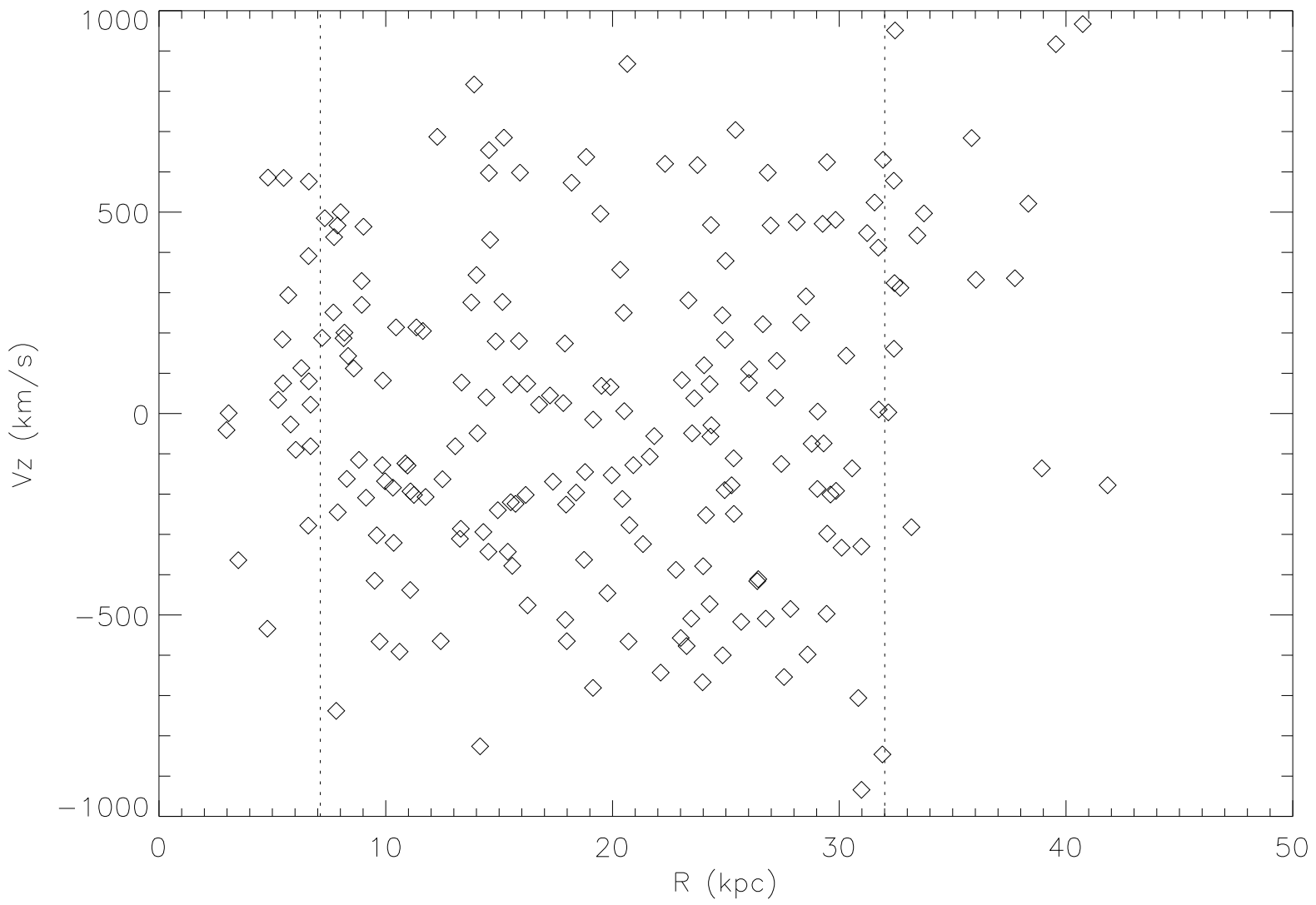

Fig. 5.- The data pairs $\left[R_{i}, v_{z i}\right]$ from Cohen and Ryzhov (1997) that we use to construct a uniform dataset. The data outside the survey radii (dotted lines) are excluded. 

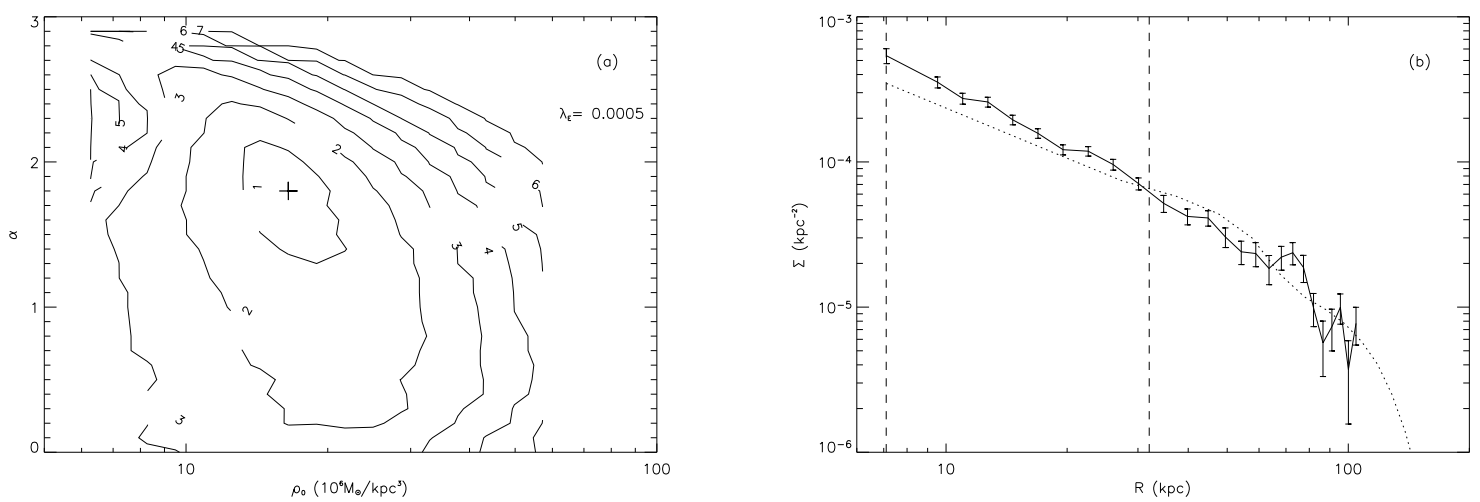

Fig. 6.- Fitting the kinematic data $\left[R_{i}, v_{z i}\right]$ on GCs in M87 to a power-law potential. This fit is based only on the 161 GCs between 7 and $32 \mathrm{kpc}$ with radial velocities. (a) Contour plot of $Q_{\max }(\mathbf{X})$ as a function of $\mathbf{X}=\left\{\rho_{0}, \alpha\right\}$. The plus sign marks the best-fit model, which gives the maximum $Q_{\max }(\mathbf{X})$. The contours are $n-\sigma$ away from the peak. (b) Surface number density profiles. The derived $\Sigma(R)$ (dotted line) is consistent with the observed surface number density $\Sigma_{0}(R)$ (solid line), even though it was not used in the fit (compare Figure 7, where it is used). The surface densities are normalized so that the total number within the range $7-110 \mathrm{kpc}$ is unity. The kinematic survey limits are marked by dashed lines. 

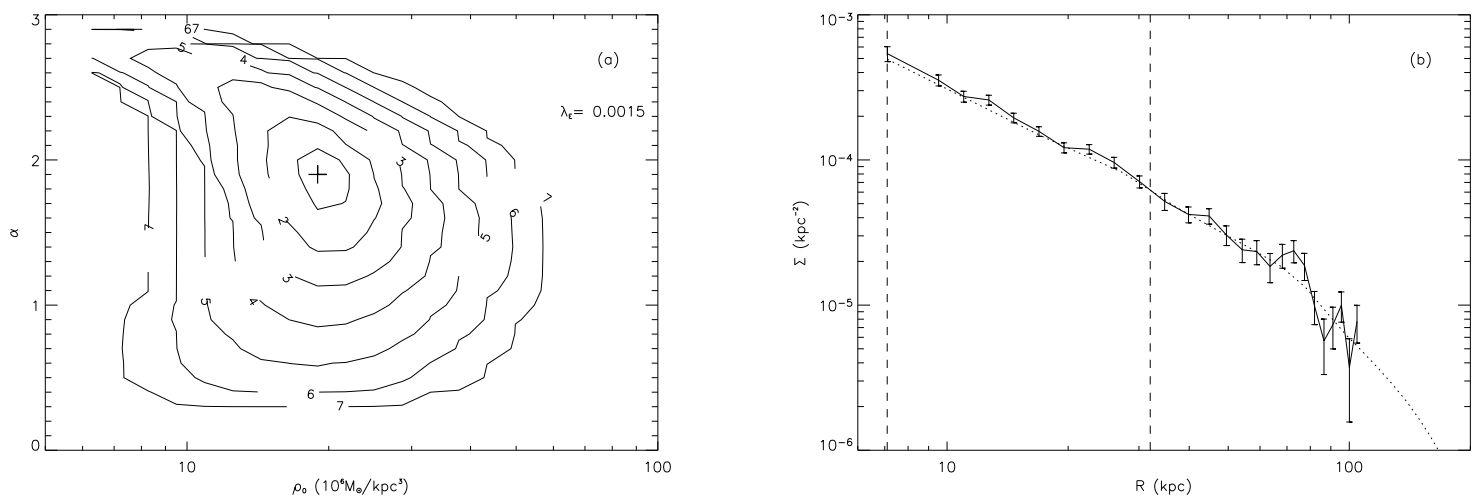

Fig. 7.- Fitting the kinematic data $\left[R_{i}, v_{z i}\right]$ and the observed surface number density $\Sigma_{0}(R)$ to a power-law potential. (a) The same as Figure 6 a except that we also fit $\Sigma_{0}(R)$, i.e., we maximize $Q^{\prime}(\mathbf{X}, \mathbf{W})$ (eq. 36) rather than $Q(\mathbf{X}, \mathbf{W})$ (eq. 33). (b) The derived surface number density (dotted line) from maximizing $Q^{\prime}(\mathbf{X}, \mathbf{W})$, compared to the observed $\Sigma_{0}(R)$ (solid line). 


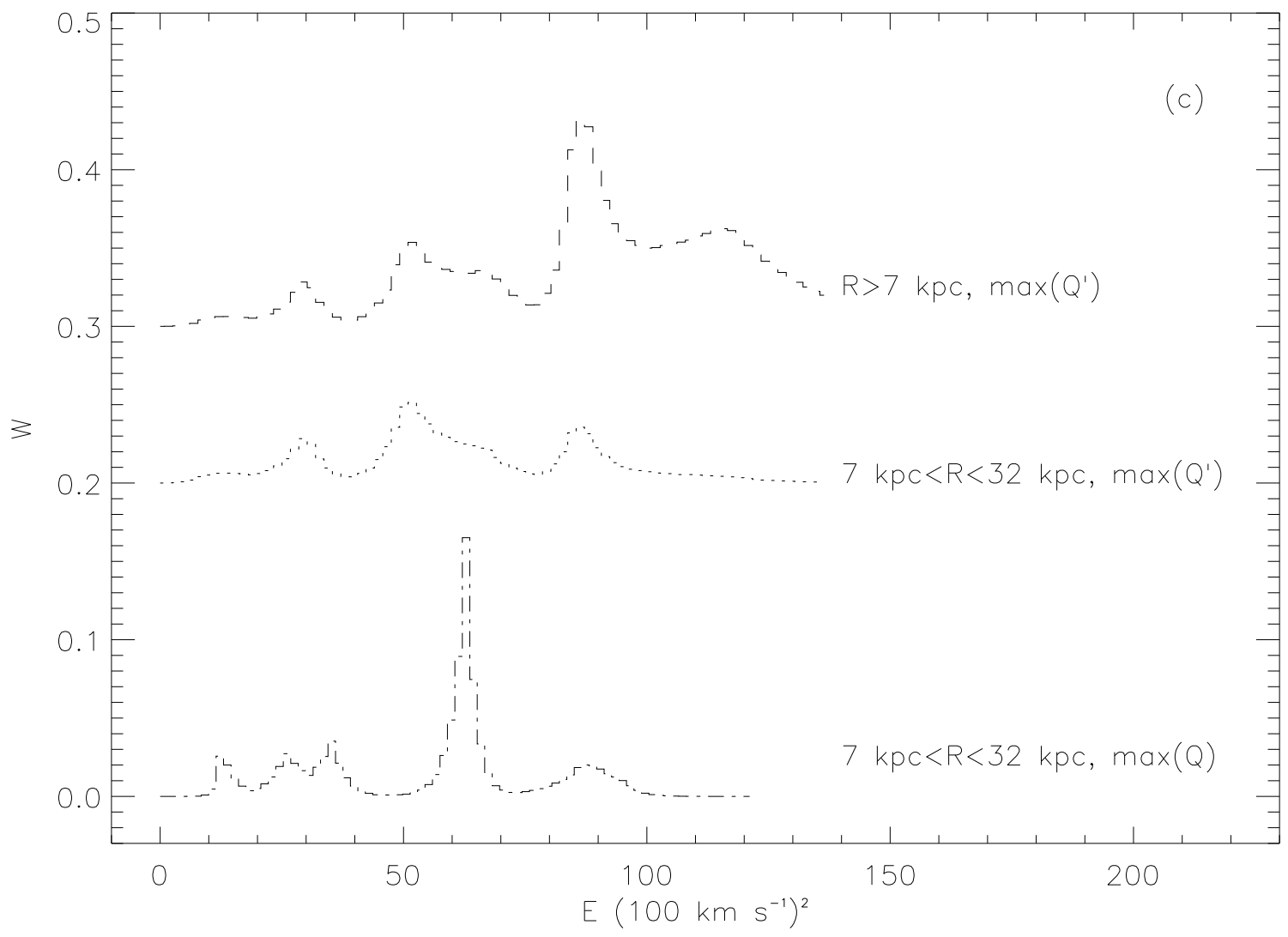

Fig. 8.- Energy distribution with the isotropic assumption: the energy weights $\mathbf{W}$ as determined by fitting the kinematic data alone, as in Figure 6 (dash-dotted line) and both the kinematic data and the surface density profile, as in Figure 7 (dotted line and dashed line). The lower two lines refer to the energy distributions of GCs within projected radius $R=7-32 \mathrm{kpc}$ and the upper line to all GCs with $R \geq 7 \mathrm{kpc}$. 

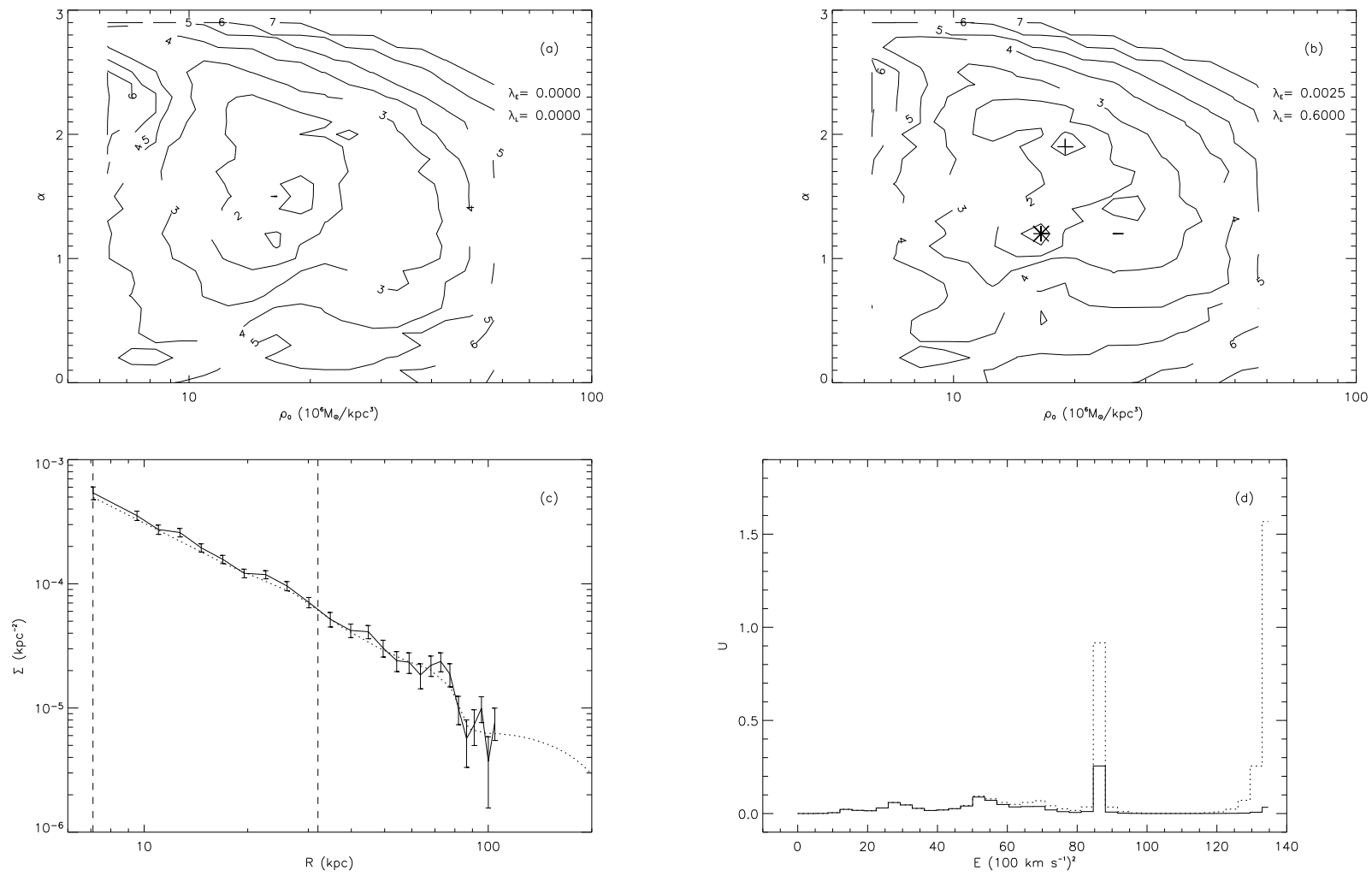

Fig. 9.- Fitting the kinematic data $\left[R_{i}, v_{z i}\right]$ and the observed surface number density $\Sigma_{0}(R)$ to a power-law potential without assuming isotropy of the DF. (a) The same as Figure 7a except for anisotropic models without regularization. (b) The same as Figure 9a except for anisotropic models with natural smoothing parameters. The plus and asterisk mark the best-fit model and a secondary peak, respectively. (c) The same as Figure 7b except for the best-fit anisotropic model with natural smoothing parameters. (d) Energy distribution of GCs within projected radius $R=7-32 \mathrm{kpc}$ (solid line) and with $R \geq 7 \mathrm{kpc}$ (dotted line). 

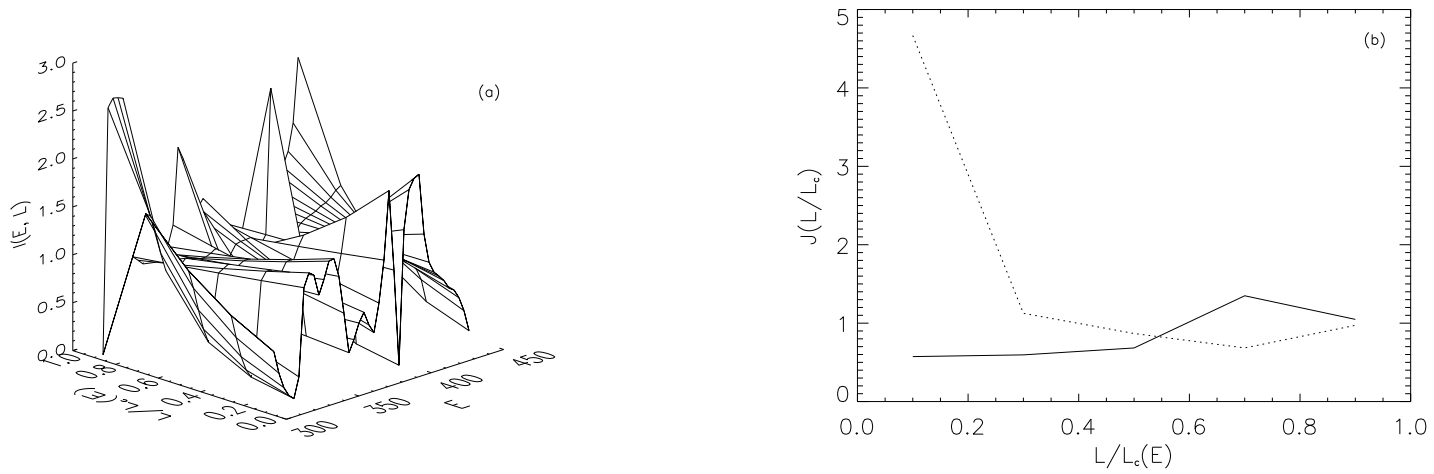

Fig. 10.- Indicators of anisotropy for $\lambda_{E}, \lambda_{L}=0.005$. (a) $I(E, L)$ (eq. 43). (b) $J\left(L / L_{c}\right)$ (eq. 45) for the best-fit model and at the secondary peak (see Figure $9 \mathrm{~b}$ ). The behavior of the indicators suggests that the GCs may either prefer circular orbits (high angular momentum) or radial orbits (low angular momentum), depending on the mass distribution model of the galaxy. 

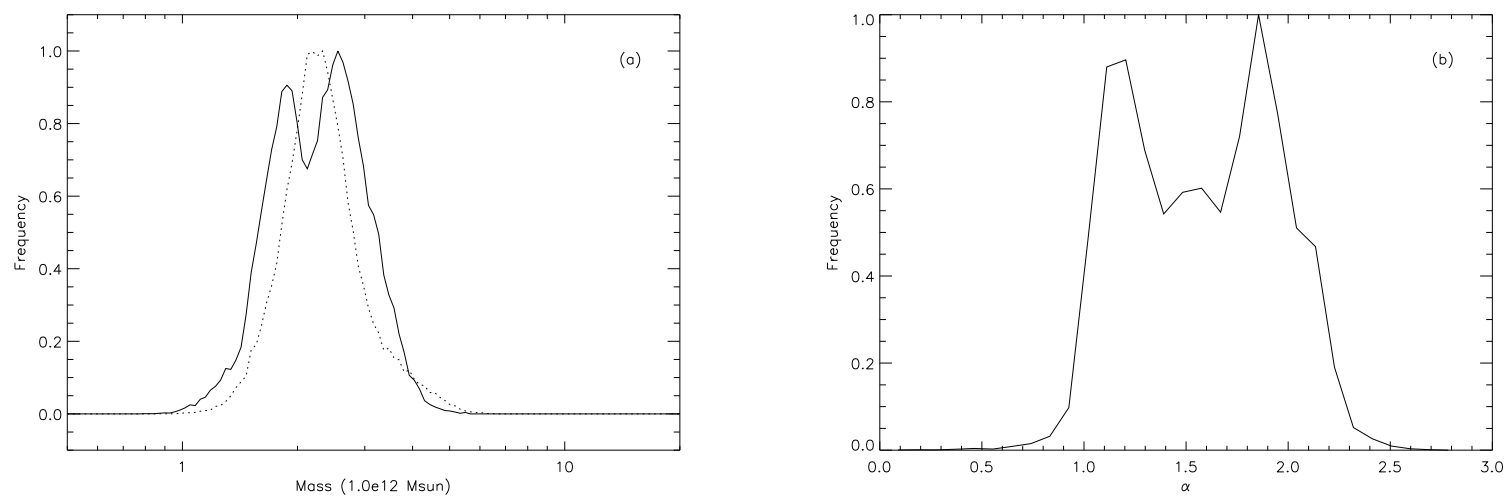

Fig. 11.- Distribution of $M(r=32 \mathrm{kpc})$ and $\alpha$ for likelihood distribution of Figure 9b. (a) The probability distribution of $M(r=32 \mathrm{kpc})$ for the power-law (solid line) and NFW models (dotted line). (b) Probability function of $\alpha$ for the power-law models. It gives $\alpha=1.6 \pm 0.4$. 

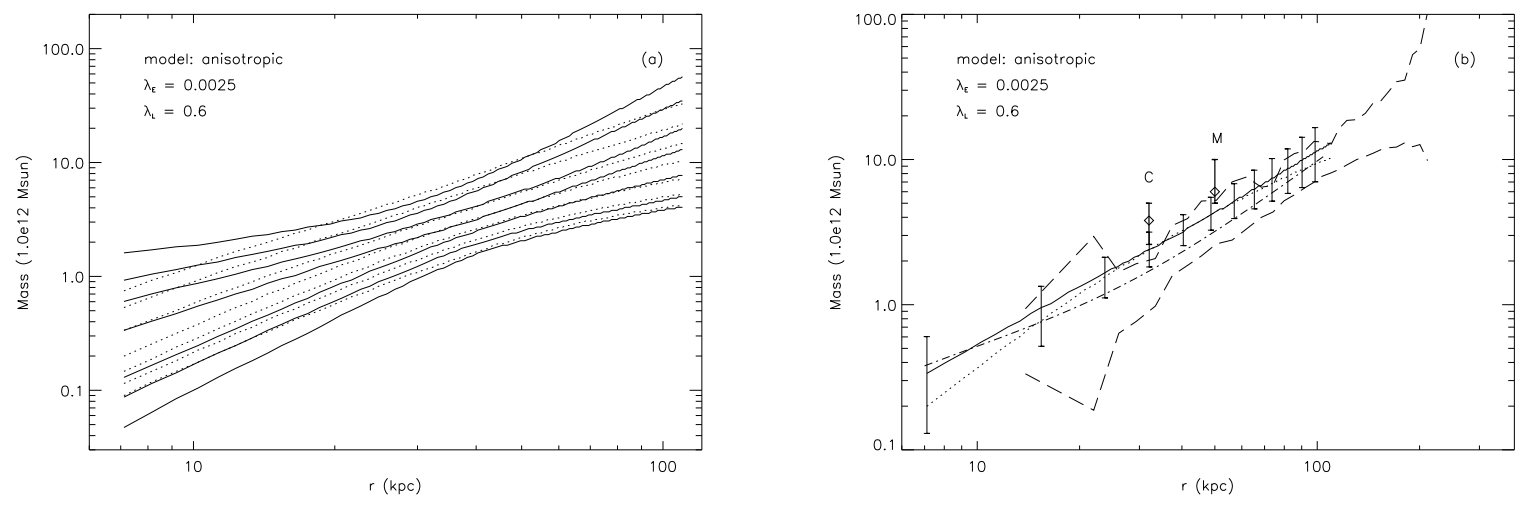

Fig. 12.- Estimates of $M(r)$, derived from Monte Carlo points laid down on Figure 9b. (a) The probability distribution of $M(r)$. The lines show the median and 1,2,3- $\sigma$ errors which enclose the regions with the cumulative probabilities of $68.3 \%, 95.4 \%, 99.7 \%$ for the powerlaw models (solid lines) and the NFW models (dotted lines). (b) The solid and dotted lines show the estimates for $M(r)$ by assuming power-law and NFW density profiles, respectively. The error bars indicate the uncertainties in the mass estimates, which are similar for both models. The other models are: Merritt \& Tremblay (1993) (marked by "M"), Cohen and Ryzhov (1997) (marked by "C"), upper and lower limits from X-ray observation by Nulsen \& Böhringer (1995) (long dashed lines), McLaughlin (1999b) (dot-dashed line). 


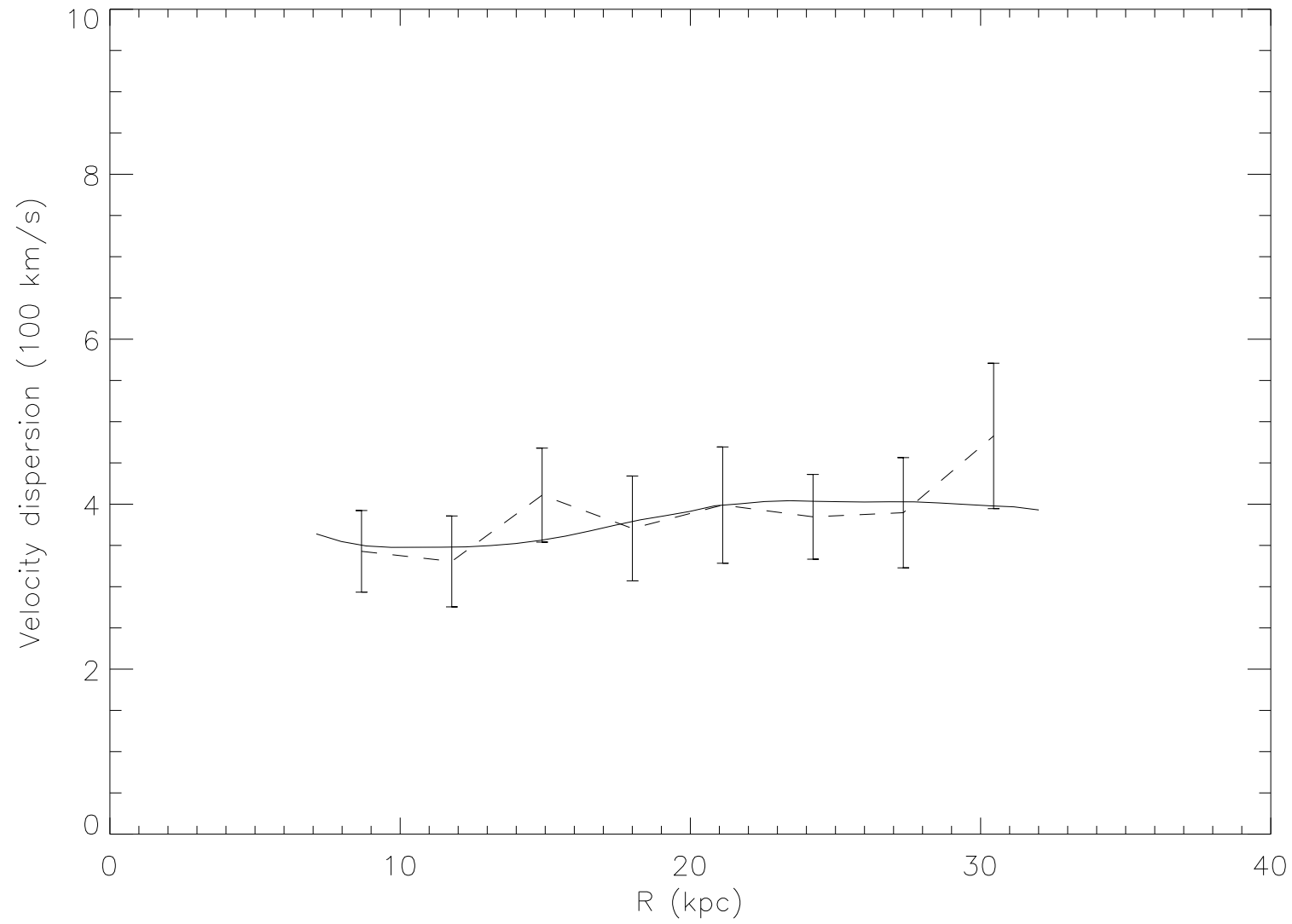

Fig. 13. - Velocity dispersion. The line is derived from the best-fit anisotropic power-law model, which is consistent with the observation (solid line with error bars). 